\title{
Gravitational mechanisms to self-tune the cosmological constant: obstructions and ways forward
}

\author{
Florian Niedermann ${ }^{1, *}$ and Antonio Padilla ${ }^{1, \dagger}$ \\ ${ }^{1}$ School of Physics and Astronomy, University of Nottingham, Nottingham NG7 2RD, UK
}

(Dated: November 16, 2017)

\begin{abstract}
Gravitational models of self-tuning are those in which vacuum energy has no observable effect on spacetime curvature, even though it is a priori unsuppressed below the cut-off. We complement Weinberg's no go theorem by studying field theoretic completions of self-adjustment allowing for broken translations as well as other generalisations, and identify new obstructions. Our analysis uses a very general Källén-Lehmann spectral representation of the exchange amplitude for conserved sources of energy-momentum and exploits unitarity and Lorentz invariance to show that a transition from self-tuning of long wavelength sources to near General Relativity on shorter scales is generically not possible. We search for novel ways around our obstructions and highlight two interesting possibilities. The first is an example of a unitary field configuration on anti-de Sitter space with the desired transition from self-tuning to GR. A second example is motivated by vacuum energy sequestering.
\end{abstract}

\section{INTRODUCTION}

The cosmological constant problem [1-5] follows automatically from our "best" model of Nature at low energies, in which matter is described by a local quantum field theory (QFT) minimally coupled to a gravitational sector described by classical General Relativity (GR). In the absence of a suitable symmetry mechanism, virtual particles endow the vacuum with an energy density scaling like the fourth power of the effective field theory cut-off. In GR, this vacuum energy gravitates like a cosmological constant, curving the spacetime geometry even in vacuum. Cosmological observations constrain the net cosmological constant to be no greater than the dark energy scale, $(\mathrm{meV})^{4}$, at least sixty orders of magnitude below the theoretical estimate based on a TeV scale cut-off, beyond which new symmetries may emerge. The precise details of this tuning are extremely sensitive to the small print of high energy physics, in complete violation of the naturalness criteria [6]. Because naturalness plays such an important role in many aspects of particle physics [7], its most notable failure, the cosmological constant problem, is generally regarded as one of the most important problems in Theoretical Physics.

The scarcity of viable proposals for solving the cosmological constant problem has led some to abandon naturalness, and seek anthropic explanations within a scannable landscape of vacua $[3,8]$. Nevertheless, there do exist natural alternatives to this, most notably vacuum energy sequestering [9-15](more on that later). In this letter we are interested in so-called self-tuning, or self-adjusting proposals, in which the vacuum energy is a priori unsuppressed below the cut-off, but has no significant observable effect on the spacetime curvature thanks to the adjustment of new fields. Such scenarios are famously forbidden by Weinberg's no go theorem [2], at least if we assume a local kinetic sector and translational invariance of the vacuum solution. If the latter assump- tion is relaxed, self-tuning can be possible, as in the socalled Fab Four scenario [16-18], although the presence of a light scalar field in the gravitational interaction poses potential problems for short distance phenomenology.

Self-tuning is also realised in the $5 \mathrm{D}$ braneworld set-up of [19], at the price of introducing a singular bulk. This singularity is tamed in a recent proposal [20], although it remains to be seen whether it yields a healthy perturbative description with viable phenomenology. Moreover, much of the past effort had its focus on 6D braneworld models where the vacuum energy curves the bulk into a cone. However, whilst the finite volume proposal $[21,22]$ relies on a radiatively unprotected parameter tuning $[23,24]$, the infinite volume realisation $[25,26]$ either suffers from a ghost instability or lacks a $4 \mathrm{D}$ gravity regime [27-30]. In fact, the pathology encountered in the latter case will fall in the class of new obstructions discussed here.

The purpose of this work is to identify new obstructions to self-tuning that complement Weinberg's approach and look for some novel ways in which we might overcome them. By self-tuning, here we really mean the absence of a geometrical response in spacetime to sources of infinite wavelength, even when localised sources gravitate normally, as required by short distance gravity tests. By this definition, we do not consider the possibility of self-tuning by scale invariance, in which the ratio of the field theory masses and the scale of gravity runs to zero, already captured by Weinberg's analysis [2], and incompatible with our low energy Universe, owing to the observed particle masses relative to the Planck scale.

Our approach to the self-tuning question is one which incorporates a complete field theoretic description, allowing for configurations that break translation invari- 
ance and have non-local kinetic operator, ${ }^{1}$ in constrast to Weinberg, and factoring in constraints from observational tests of GR up to solar system scales [33]. To this end, we consider the exchange amplitude for two conserved sources of energy momentum in a background Minkowski spacetime, mediated by both single and multiparticle states up to spin-2. We use a Källén-Lehmann (KL) spectral representation [34, 35] to capture the fully quantum corrected exchange amplitude that is linear in each source. We assume that the intermediate states couple linearly to sources and that the free field propagators are of their canonical form, compatible with unitarity and Lorentz invariance. By further imposing unitarity through the positivity of the spectral density we are able to show that generically one cannot pass from a selftuning regime for sources of infinite wavelength to one in which we recover GR to sufficient precision for localised sources. We then look for novels ways in which we can get around our obstructions.

Our analysis can be extended to a background de Sitter spacetime with the same generic results but not to a background anti-de Sitter. Indeed, one can find explicit examples of linearised field configurations in antide Sitter that self-tune at large distances but recover GR on shorter scales with the correct tensor structure. We identify a second way around our obstructions motivated by vacuum energy sequestering [9-15], being of greater phenomenological interest. There we find that the structure of the free field propagator is not always canonical, but exhibits features reminiscent of the decapitiation scenario [36]. A third possible way around involves so-called screening mechanisms [37-43] which rely on non-linear couplings to the source.

\section{OBSTRUCTIONS TO SELF-TUNING IN MINKOWSKI SPACETIME}

Consider the exchange amplitude for two conserved sources, $T_{\mu \nu}$ and $T_{\mu \nu}^{\prime}$ on a Minkowski background, mediated by both single and multi-particle states up to spin2. Since we assume linear couplings to conserved sources, spin-1 states cannot couple to the source, so we only need to consider intermediate states of spin- 0 and 2 . Assuming Lorentz invariance and unitarity, we can express the

1 The only requirement is that the kinetic operator admits a Källén-Lehmann spectral decomposition. The braneworld inspired propagator discussed in [31] provides a prototypical example (see also [32]). amplitude, using a spectral representation, as

$$
\begin{aligned}
\mathcal{A}=\bar{\rho}_{2} \overline{\mathcal{A}}_{2}+\bar{\rho}_{0} \overline{\mathcal{A}}_{0}+ \\
\int_{0^{+}}^{\infty} \mathrm{d} s \rho_{2}(s) \mathcal{A}_{2}(s)+\int_{0^{+}}^{\infty} \mathrm{d} s \rho_{0}(s) \mathcal{A}_{0}(s),
\end{aligned}
$$

where we have included the exchange of a single massless spin-2 state with coupling $\bar{\rho}_{2}$, massive spin-2 states of mass $s$ and spectral density $\rho_{2}(s)$, a massless spin- 0 state with coupling $\bar{\rho}_{0}$, as well as a spin-0 states of mass $s$ and spectral density $\rho_{0}(s)$. The form of the free field propagators are those of canonical fields with the appropriate mass and spin, fixed by Lorentz invariance and unitarity. The corresponding one-particle exchange amplitudes are specified in Eqs. (7) and (9) for spin-2 (after setting $\kappa=0$ ), whereas for spin-0 we have

$$
\begin{aligned}
\overline{\mathcal{A}}_{0}=\int \mathrm{d}^{4} x \sqrt{-\bar{g}} T^{\prime} \frac{1}{-\bar{\square}} T, \\
\mathcal{A}_{0}(s)=\int \mathrm{d}^{4} x \sqrt{-\bar{g}} T^{\prime} \frac{1}{-\bar{\square}+s} T,
\end{aligned}
$$

where $\sqrt{-\bar{g}}=1$ in the Minkowski case. The Green's function acting on a tensor of arbitrary rank is given by $\left(\frac{1}{-\bar{\square}+\mu^{2}} J_{\cdots}^{\cdots}\right)(x)=\int \mathrm{d}^{4} y G_{\mu}(x, y) J_{\cdots}^{\cdots}(y)$, where $\left(-\bar{\square}_{x}+\right.$ $\left.\mu^{2}\right) G_{\mu}(x, y)=\delta(x-y)$. In general, the precise form of these Green's functions will not be important for our discussion, although we will state certain important properties where appropriate. Unitarity requires us to assume positivity of the spectral densities, i.e. $\bar{\rho}_{2}, \bar{\rho}_{0}, \rho_{2}(s), \rho_{0}(s) \geq 0$.

If this theory is to "self-tune" along the lines described in the introduction, the amplitude should vanish in the presence of an infinite wavelength vacuum energy source, $T_{\mu \nu}=-V_{v a c} \eta_{\mu \nu}$, and a localised probe, $T_{\mu \nu}^{\prime}$, or in other words

$$
\frac{2}{3} \bar{\rho}_{2} \frac{1}{-\bar{\square}} 1-4 \bar{\rho}_{0} \frac{1}{-\bar{\square}} 1-4 \int_{0^{+}}^{\infty} \mathrm{d} s \rho_{0}(s) \frac{1}{s} 1=0,
$$

where the convolution of the Green's function with unity is understood, and we have used the fact that for $s \neq 0$, $\frac{1}{-\square+s} 1=\frac{1}{s}$. Moreover, the massless Green's function was assumed to be Lorentz invariant ensuring the vanishing of the transverse-tracefree part of the energymomentum tensor $T_{\mu \nu}^{(\mathrm{TT})}$ for a constant vacuum energy source. If we operate on Eq. (3) with $\bar{\square}$, we obtain the "self-tuning" relation: $\bar{\rho}_{2}=6 \bar{\rho}_{0}$.

In contrast, for localised sources at shorter wavelength, we demand close agreement with GR in order to recover its experimental success [33], specifically

$$
\mathcal{A} \rightarrow \frac{1}{M_{\mathrm{Pl}}^{2}} \int \mathrm{d}^{4} x\left[T^{\prime \mu \nu} \frac{1}{-\bar{\square}} T_{\mu \nu}-\frac{1}{2}(1-\epsilon) T^{\prime} \frac{1}{-\bar{\square}} T\right],
$$

as $-\bar{\square} \rightarrow \infty$, with $|\epsilon| \lesssim 10^{-5}$. We use Eqs. (2), (8) and (10) to express $\mathcal{A}$ in terms of the spectral densities and 
sources. The above condition then imposes two ultraviolet constraints, which can be obtained by comparing the tensor $\left(\propto T^{\mu \nu}\right)$ and scalar $(\propto T)$ contributions to $\mathcal{A}$ independently. We express them in momentum space as

$$
\begin{gathered}
\frac{\bar{\rho}_{2}}{x}+\int_{0^{+}}^{\infty} \mathrm{d} s \frac{\rho_{2}(s)}{x+s} \rightarrow \frac{1}{M_{\mathrm{Pl}}^{2}} \frac{1}{x} \\
\frac{1}{3} \int_{0^{+}}^{\infty} \mathrm{d} s \frac{\rho_{2}(s)}{x+s}+2 \frac{\bar{\rho}_{0}}{x}+2 \int_{0^{+}}^{\infty} \mathrm{d} s \frac{\rho_{0}(s)}{x+s} \rightarrow \frac{\epsilon}{M_{\mathrm{Pl}}^{2}} \frac{1}{x}
\end{gathered}
$$

as $x \equiv p_{\mu} p^{\mu} \rightarrow \infty$. Now, thanks to positivity of the spectral densities, we have that $\frac{\bar{\rho}_{2}}{x}, \frac{\bar{\rho}_{0}}{x}, \int_{0^{+}}^{\infty} \mathrm{d} s \frac{\rho_{2}(s)}{x+s}$, $\int_{0^{+}}^{\infty} \mathrm{d} s \frac{\rho_{0}(s)}{x+s} \geq 0, \forall x \geq 0$, and so from Eq. (5b) we infer that $\bar{\rho}_{0}, x \int_{0^{+}}^{\infty} \mathrm{d} s \frac{\rho_{2}(s)}{x+s}, x \int_{0^{+}}^{\infty} \mathrm{d} s \frac{\rho_{0}(s)}{x+s} \lesssim|\epsilon| / M_{\mathrm{Pl}}^{2}$ at large $x$. By the condition in Eq. (5a), we now find that $\bar{\rho}_{2} \sim 1 / M_{\mathrm{Pl}}^{2}$. The ultra-violet conditions $\bar{\rho}_{2} \sim 1 / M_{\mathrm{Pl}}^{2}$ and $\bar{\rho}_{0} \lesssim|\epsilon| / M_{\mathrm{Pl}}^{2}$ are then in contradiction with the "selftuning" condition, $\bar{\rho}_{2}=6 \bar{\rho}_{0}$, obtained in the infra-red - we do not seem able to self-tune at large wavelength and recover GR at short wavelength. Modulo our initial assumptions, this represents a significant obstruction to self-tuning on a Minkowski background.

\section{EXCHANGE OF A SINGLE GRAVITON IN MAXIMALLY SYMMETRIC SPACE}

Before extending our analysis to (anti-)de Sitter spaces, we consider the amplitude for the exchange of a single graviton between two conserved sources, $T_{\mu \nu}$ and $T_{\mu \nu}^{\prime}$, on a maximally symmetric spacetime, with curvature $\kappa$. In general, it is given by

$$
\mathcal{A}=\frac{1}{2} \int \mathrm{d}^{4} x \sqrt{-\bar{g}} h_{\mu \nu} T^{\prime \mu \nu},
$$

where $\bar{g}_{\mu \nu}$ is the background metric, and $h_{\mu \nu}$ the graviton fluctuation due to a source $T_{\mu \nu}$.

Solving the linearized Einstein equations, we find $\left.\mathcal{A}\right|_{m^{2}=0}=\overline{\mathcal{A}}_{2} / M_{\mathrm{Pl}}^{2}$, where

$$
\begin{aligned}
\overline{\mathcal{A}}_{2}=-\int \mathrm{d}^{4} x \sqrt{-\bar{g}}\left\{T_{(\mathrm{TT})}^{\prime \mu \nu}\right. & \left.\frac{1}{\square-2 \kappa}\right|_{t} T_{\mu \nu}^{(\mathrm{TT})} \\
& \left.-\left.\frac{1}{6} T^{\prime} \frac{1}{\bar{\square}+4 \kappa}\right|_{s} T\right\} .
\end{aligned}
$$

Here,

$T_{\mu \nu}^{(\mathrm{TT})}=T_{\mu \nu}+\frac{1}{3}\left[\bar{\nabla}_{\mu} \bar{\nabla}_{\nu}-\bar{g}_{\mu \nu}(\bar{\square}+3 \kappa)\right]\left(\left.\frac{1}{\bar{\square}+4 \kappa}\right|_{s} T\right)$,

and $\left.\frac{1}{\square-\mu^{2}}\right|_{s, t}$ is the inverse of the operator $\square-\mu^{2}$ acting on scalars (s) and tensors (t), respectively. The corresponding Green's functions respect Lorentz invariance and satisfy $\left.\frac{1}{\square-\mu^{2}}\right|_{s} 1=-\frac{1}{\mu^{2}}$, for $\mu^{2} \neq 0$. For $\mu^{2}=0$ and on a Minkowski background, we will also need the relation $\left.\partial_{\mu} \partial_{\nu} \frac{1}{\bar{\square}}\right|_{s} 1=\frac{1}{4} \eta_{\mu \nu}$, as motivated by Lorentz invariance.

For localised conserved sources, we can manipulate this expression into a more familiar form

$$
\begin{aligned}
\overline{\mathcal{A}}_{2}=-\int \mathrm{d}^{4} x & \sqrt{-\bar{g}}\left\{\left.T^{\prime \mu \nu} \frac{1}{\bar{\square}-2 \kappa}\right|_{t} T_{\mu \nu}\right. \\
& \left.-\frac{1}{2} T^{\prime}\left[\left.\frac{\frac{1}{2}}{\bar{\square}-2 \kappa}\right|_{s}+\left.\frac{\frac{1}{2}}{\bar{\square}+6 \kappa}\right|_{s}\right] T\right\} .
\end{aligned}
$$

For $m^{2} \neq 0,2 \kappa,{ }^{2}$ we obtain $\mathcal{A}_{m^{2} \neq 0,2 \kappa}=\mathcal{A}_{2}\left(m^{2}\right) / M_{\mathrm{Pl}}^{2}$, where

$$
\begin{gathered}
\mathcal{A}_{2}\left(m^{2}\right)=-\int \mathrm{d}^{4} x \sqrt{-\bar{g}}\left\{\left.T_{(\mathrm{TT})}^{\prime \mu \nu} \frac{1}{\bar{\square}-2 \kappa-m^{2}}\right|_{t} T_{\mu \nu}^{(\mathrm{TT})}\right. \\
\left.-\left.\frac{\kappa}{3\left(2 \kappa-m^{2}\right)} T^{\prime} \frac{1}{\bar{\square}+4 \kappa}\right|_{s} T\right\} .
\end{gathered}
$$

This formula holds in all cases, although for two nonlocalised, but constant, vacuum energy sources care must be taken to take the $\kappa=0$ limit only after identifying $\frac{1}{\square+4 \kappa}$ with $\frac{1}{4 \kappa}$.

For two localised conserved sources, we can once again manipulate this formula into the following form,

$$
\begin{gathered}
\mathcal{A}_{2}\left(m^{2}\right)=-\int \mathrm{d}^{4} x \sqrt{-\bar{g}}\left\{\left.T^{\prime \mu \nu} \frac{1}{\bar{\square}-2 \kappa-m^{2}}\right|_{t} T_{\mu \nu}\right. \\
\left.-\frac{1}{2} T^{\prime}\left[\frac{1 / 2}{\bar{\square}-2 \kappa-m^{2}}+\frac{\kappa-m^{2} / 6}{\kappa-m^{2} / 2} \frac{1 / 2}{\bar{\square}+6 \kappa-m^{2}}\right]_{s} T\right\} .
\end{gathered}
$$

Notice the absence of the vDVZ discontinuity $[49,50]$ as $m^{2} \rightarrow 0$ for $\kappa \neq 0[44,51]$.

\section{OBSTRUCTIONS TO SELF-TUNING IN DE SITTER AND ANTI-DE SITTER SPACETIME}

We shall now attempt to extend our analysis to a background de Sitter or anti-de Sitter spacetime, with curvature $\kappa$. Of course, we normally associate the notion of self-tuning with Minkowski vacua, although we can easily extend its notion to other maximally symmetric vacua by requiring that vacuum energy sources do not gravitate. The so-called Fab 5 models are an example of this in a de Sitter background [52]. In any event, the exchange

2 The partially massless case [45] with $m^{2}=2 \kappa$ is not considered here since it has been argued to contain an infinitely strongly coupled helcility-0 mode in violation of perturbative unitarity [46-48]. 
amplitude for conserved sources, analogous to Eq. (1), is given by

$$
\begin{aligned}
\mathcal{A}=\bar{\rho}_{2} \overline{\mathcal{A}}_{2}+\bar{\rho}_{0} \overline{\mathcal{A}}_{0}+ \\
\int_{2 \kappa^{+}}^{\infty} \mathrm{d} s \rho_{2}(s) \mathcal{A}_{2}(s)+\int_{s_{0}}^{\infty} \mathrm{d} s \rho_{0}(s) \mathcal{A}_{0}(s),
\end{aligned}
$$

with the respective ( $\kappa$-dependent) amplitudes defined in the previous section and Eq. (2). Although this amplitude is the natural extension of the Minkowski one discussed before, we hesitate to interpret it as a KL representation of the full quantum corrected amplitude on account of the non-trivial asymptotic structure (see [5355] for a discussion of KL representations in de Sitter and anti-de Sitter). The lower limits on the integrals are fixed by the stability of the theory. In particular, perturbative unitarity imposes the constraint on the spin-2 limit $s>2 \kappa[45-48,56]$. In anti-de Sitter $(\kappa<0)$, we impose the Breitenlohner-Freedman bound on the scalar masses, $s_{0}=4 \kappa[57]^{3}$. We are not aware of an analogous bound in de Sitter $(\kappa>0)$, so for now we conservatively impose $s_{0}>0$.

Again, for "self-tuning" we demand that the amplitude should vanish for a vacuum energy source, $T_{\mu \nu}=$ $-V_{v a c} \bar{g}_{\mu \nu}$, that is

$$
\frac{\bar{\rho}_{2}}{6 \kappa}+4 \bar{\rho}_{0} \frac{1}{-\square} 1-\frac{1}{3} \int_{2 \kappa^{+}}^{\infty} \mathrm{d} s \frac{\rho_{2}(s)}{s-2 \kappa}+4 \int_{s_{0}}^{\infty} \mathrm{d} s \frac{\rho_{0}(s)}{s}=0 .
$$

Acting with $\bar{\square}$ on this equation sets $\bar{\rho}_{0}=0$. In the ultraviolet, we again demand that Eq. (4) holds, implying a modified version of Eqs. (5) (after performing the same steps as before),

$$
\begin{gathered}
\frac{\bar{\rho}_{2}}{x}+\int_{2 \kappa^{+}}^{\infty} \mathrm{d} s \frac{\rho_{2}(s)}{x+s} \rightarrow \frac{1}{M_{\mathrm{Pl}}^{2}} \frac{1}{x} \\
\frac{1}{3} \int_{2 \kappa^{+}}^{\infty} \mathrm{d} s \frac{\rho_{2}(s)}{x+s}+\frac{2 \kappa}{3} \int_{2 \kappa^{+}}^{\infty} \mathrm{d} s \frac{\rho_{2}(s)}{x+s} \frac{1}{s-2 \kappa} \\
+2 \frac{\bar{\rho}_{0}}{x}+2 \int_{s_{0}}^{\infty} \mathrm{d} s \frac{\rho_{0}(s)}{x+s} \rightarrow \frac{\epsilon}{M_{\mathrm{Pl}}^{2}} \frac{1}{x}
\end{gathered}
$$

as $x \rightarrow \infty$. For a de Sitter background $(\kappa>0)$, at large $x$, positivity of the spectral densities guarantees the positivity of each individual term in Eq. (13b). It then follows that $x \int_{2 \kappa}^{\infty} \mathrm{d} s \frac{\rho_{2}(s)}{x+s} \lesssim|\epsilon| / M_{\mathrm{Pl}}^{2}$, enabling us to

\footnotetext{
3 Since the range of integration for the massive states in AdS passes through zero, with massless states included explicitly elsewhere via $\bar{\rho}_{0}$ and $\bar{\rho}_{2}$, we shall set $\rho_{0}^{A d S}(0)=\rho_{2}^{A d S}(0)=0$. This technical point is included only in the interests of rigour - it will play no role in our analysis.
}

infer, using Eq. (13a), that $\bar{\rho}_{2} \sim 1 / M_{\mathrm{Pl}}^{2}$. Furthermore, as $x \rightarrow \infty$, we may also infer that

$$
\begin{aligned}
\frac{|\epsilon|}{M_{\mathrm{Pl}}^{2}} & \gtrsim \quad \kappa \int_{2 \kappa^{+}}^{\infty} \mathrm{d} s \frac{\rho_{2}(s)}{s-2 \kappa} \frac{1}{1+s / x} \\
& \underset{\text { positivity }}{\gtrsim} \kappa \int_{2 \kappa^{+}}^{s_{*}} \mathrm{~d} s \frac{\rho_{2}(s)}{s-2 \kappa} \frac{1}{1+s / x} \\
\underset{x \gg s_{*}}{\sim} & \kappa \int_{2 \kappa^{+}}^{s_{*}} \mathrm{~d} s \frac{\rho_{2}(s)}{s-2 \kappa}>0
\end{aligned}
$$

where $s_{*}$ is some arbitrarily large, but finite, constant. We emphasize that this only applies in de Sitter space - no such inferences can be made on an anti-de Sitter background $(\kappa<0)$.

Staying with de Sitter space, let us consider the infrared condition in Eq. (12). By plugging in $\bar{\rho}_{2} \sim 1 / M_{\mathrm{Pl}}^{2}$, $\bar{\rho}_{0}=0$ and using the inequality (14), we obtain ${ }^{4}$

$$
24 \kappa M_{\mathrm{Pl}}^{2} \int_{s_{0}}^{\infty} \mathrm{d} s \frac{\rho_{0}(s)}{s} \sim-1+\mathcal{O}(\epsilon),
$$

which for $\kappa>0$ is in contradiction with the positivity of $\rho_{0}(s)$. This extends the results of the previous section to de Sitter space.

\section{THE ANTI-DE SITTER LOOPHOLE}

In the previous section, we saw how our conclusions could not trivially be extended to anti-de Sitter space. This raises the question: is there an anti-de Sitter loophole we can exploit? It turns out that there is. For an explicit example, consider the following two-parameter family of spectral densities on a background anti-de Sitter space: $\bar{\rho}_{2}=\frac{1}{M_{\mathrm{P} 1}^{2}}\left(1-\alpha_{2}\right), \rho_{2}(s)=\frac{\alpha_{2}}{M_{\mathrm{Pl}}^{2}} \delta\left(s-\mu_{2}\right)$, $\bar{\rho}_{0}=0, \rho_{0}(s)=\frac{\alpha_{0}}{6 M_{\mathrm{Pl}}^{2}} \delta\left(s-\mu_{0}\right)$, with couplings

$$
\alpha_{2}=\frac{\mu_{0}}{\mu_{2}} \frac{\mu_{2}-2 \kappa}{\mu_{0}+4 \kappa}, \quad \alpha_{0}=\frac{-\mu_{0}}{\mu_{0}+4 \kappa} .
$$

This describes a massless graviton as well as a single massive graviton and scalar of mass squared $\mu_{2} \in\left(2 \kappa,-\mu_{0} / 2\right]$ and $\mu_{0} \in(0,-4 \kappa)$, respectively, consistent with unitarity [45] and stability [57]. It is easy to check that this fulfils both the ultra-violet (13) and the infrared constraint (12).

\section{THE SEQUESTERING LOOPHOLE}

In vacuum energy sequestering [9-15], vacuum energy does not gravitate yet the theory recovers GR for localised sources. It is instructive to see how it gets past 4 This is true as long as the spectral density $\rho_{2}(s)$ does not exhibit
any isolated singular behaviour at infinity. 
the obstructions described in this letter. To this end, consider the effective gravitational equation of motion in the original global version of the theory $[9,10]$

$$
M_{\mathrm{Pl}}^{2} G_{\mu \nu}=T_{\mu \nu}-\frac{1}{4}\langle T\rangle g_{\mu \nu}
$$

where the spacetime average of the trace of the energy momentum source is given by $\langle T\rangle=$ $\int \mathrm{d}^{4} x \sqrt{-g} T / \int \mathrm{d}^{4} x \sqrt{-g}$. Although this particular version $^{5}$ of sequestering can only be consistent with our universe if the spacetime volume is finite, we can study the dynamics of fluctuations in a locally Minkowski frame, approximating the volume integrals as integrals over a very large but finite section of Minkowski space. As shown explictly in [14], this represents an excellent approximation especially when we are close to the maximum scale factor in the cosmological evolution. In any event, if we proceed in this way the exchange amplitude is given by $\mathcal{A}=\frac{1}{2} \int \mathrm{d}^{4} x h_{\mu \nu}\left(T^{\prime \mu \nu}-\frac{1}{4}\left\langle T^{\prime}\right\rangle \eta_{\mu \nu}\right)$, and so we find that

$$
\begin{aligned}
\mathcal{A}=\mathcal{A}_{G R} & +\frac{1}{6 M_{\mathrm{Pl}}^{2}} \int \mathrm{d}^{4} x T^{\prime} \frac{1}{-\square} T \\
& -\frac{1}{6 M_{\mathrm{Pl}}^{2}} \int \mathrm{d}^{4} x \mathrm{~d}^{4} y T(x) \delta G(x, y) T(y),
\end{aligned}
$$

where $\mathcal{A}_{G R}=\frac{1}{M_{\mathrm{Pl}}^{2}} \overline{\mathcal{A}}_{2}$ is the standard GR amplitude, and we recall that $\frac{1}{-\square} T$ denotes a convolution with the corresponding Green's function $\int \mathrm{d}^{4} y G_{0}(x, y) T(y)$. From this we also introduce the "decapitated" Green's function [36], $\delta G(x, y)=G_{0}(x, y)-\frac{1}{V} \int \mathrm{d}^{4} z G_{0}(x, z)-$ $\frac{1}{V} \int \mathrm{d}^{4} z G_{0}(z, y)+\frac{1}{V^{2}} \int \mathrm{d}^{4} z \mathrm{~d}^{4} w G_{0}(z, w)$ and $V=\int \mathrm{d}^{4} z$. This has the property that it vanishes for exactly zero momentum, i.e., it vanishes when it is convoluted with a constant $\int \mathrm{d}^{4} y \delta G(x, y)=\int \mathrm{d}^{4} x \delta G(x, y)=0$. Furthermore, at non-zero momentum it behaves like the usual canonical Green's function, $G_{0}(x, y)$. In other words, when it acts on a localised excitation $\delta T=T-\langle T\rangle$, it gives $\int \mathrm{d}^{4} y \delta G(x, y) \delta T(y)=\int \mathrm{d}^{4} y G_{0}(x, y) \delta T(y)$.

What does all this amount to? The amplitude in Eq. (18) describes the exchange of a massless particle of spin2 , a massless particle of spin-0, and a massless decapitated particle of spin-0 that happens to be ghostlike. For localised sources, the latter two contributions cancel out, yielding a well behaved GR like amplitude. The cancellation follows along similar lines to the cancellation of the brane bending mode and the conformal ghost in Randall Sundrum gravity [58-60]. In contrast, for a constant, vacuum energy source ${ }^{6}$, the decapitated ghost gets decoupled. This leaves the remaining spin- 2 and spin- 0

5 This condition can be relaxed in local formulations of the sequestering proposal $[12,14]$

6 Strictly speaking, our derivation of the amplitude in Eq. (18) is particles to combine in such a way as to force the vanishing of the amplitude, as required for self-tuning.

Clearly the amplitude Eq. (18) has an exotic field theory content, in contrast to our generic case Eq. 1, which assumed that the free field propagators took on their canonical form, and did not include the possibility of decapitated propagators as one finds in this approach to vacuum energy sequestering. A more thorough analysis of linearised theory in the various versions of vacuum energy sequestering is certainly warranted.

\section{DISCUSSION}

In this letter we have shown how difficult it is to eliminate the effect of the radiatively unstable vacuum energy contribution through "self-tuning" in a phenomenologically viable theory of gravity. By studying generic exchange amplitudes for conserved sources of energy momentum we have identified significant obstructions to self-tuning in the far infra-red and recovery of GR (to sufficient accuracy) in the ultra-violet:

Self-tuning models that admit a standard spectral representation in terms of massless and massive spin-0 and spin-2 states are generically incompatible with unitarity on both Minkowski and de Sitter backgrounds, provided the source coupling is linear.

For example, our analysis suggests that naive completions of, say, the old self-tuning brane model [19], or the "filter" proposal [61] are likely to face significant challenges from either phenomenology or unitarity.

There are ways around our obstructions, however, and we identify a couple, each of which should provide a seed of future research. For each loophole linearised selftuning can take place at low momentum even when we recover linearised GR at high momentum.

From a phenomenological perspective, the loophole suggested by vacuum energy sequester holds greatest promise. It is achieved by trading some canonical free-field propagators for decapitated ones, along the lines introduced in [36]. This connection of ideas merits further investigation and is, perhaps, the most important result to come from our work.

A second loophole occurs if the background curvature is anti-de Sitter space. Whether or not this is of interest phenomenologically remains to be seen, although we think it is unlikely. The presence of the anti-de Sitter loophole presents something of a puzzle from the point of view of any cosmological observer probing physics well

only valid down to an infra-red cut-off set by the background curvature of our finite cosmology. Nevertheless it is interesting to extend the regime of validity of our result to see, in principle at least, how the obstructions of the previous sections might be evaded. 
within the cosmological horizon, decoupled from the details of the far infra-red. This conclusion is too quick. Although the probe is a short distance one, to explore the possibility of self-tuning one must ask how it interacts with a very long wavelength source. In this sense one is not considering scattering processes that are genuinely decoupled from the infra-red. The structure of the asymptotic vacuum matters with some field configurations catastrophically destabilising the vacuum in some cases, but not others, through ghost-like excitations.

A third loophole concerns our assumption that the sources couple linearly to the states mediating the force. This cannot capture so-called screening mechanisms, such as Vainshtein [39-41], chameleons [37, 38] or symmetrons $[42,43]$, where non-linear couplings to matter play a vital role. It would be very interesting, although non-trivial, to try to incorporate these effects into our analysis. We are not aware of any self-tuning models that claim to exploit either chameleons or symmetrons in order to recover GR on the appropriate scale. The Vainshtein loophole, on the other hand, was also pointed out in Ref. [62] in the special case of a spin-2 resonance theory with non-constant mass $m^{2} \propto \square^{\alpha}(\alpha<1)$ and later used to realize self-tuning in the "degravitation" proposal in Ref. [31], however without offering a fundamental theory. The general problem with Vainshtein screening, of course, regards its regime of validity of the effective theory and whether or not it can be trusted in the regime where screening is required [63-65].

Acknowledgments: We would like to thank Christos Charmoussis, Stefan Hofmann, Nemanja Kaloper, and Peter Millington for useful discussions. F.N and A.P. are funded by an STFC Consolidated Grant. A.P is also funded by a Leverhulme Trust Research Project Grant.

* Electronic address: florian.niedermann@nottingham. ac.uk

$\dagger$ Electronic address: antonio.padilla@nottingham.ac. uk

[1] Y. B. Zeldovich, JETP Lett. 6, 316 (1967) [Pisma Zh. Eksp. Teor. Fiz. 6, 883 (1967)]; Sov. Phys. Usp. 11, 381 (1968).

[2] S. Weinberg, Rev. Mod. Phys. 61, 1 (1989).

[3] J. Polchinski, hep-th/0603249.

[4] C. P. Burgess, arXiv:1309.4133 [hep-th].

[5] A. Padilla, arXiv:1502.05296 [hep-th].

[6] G. 't Hooft, NATO Sci. Ser. B 59 (1980) 135.

[7] G. F. Giudice, In *Kane, Gordon (ed.), Pierce, Aaron (ed.): Perspectives on LHC physics* 155-178 [arXiv:0801.2562 [hep-ph]].

[8] R. Bousso, Gen. Rel. Grav. 40 (2008) 607 [arXiv:0708.4231 [hep-th]].

[9] N. Kaloper and A. Padilla, Phys. Rev. Lett. 112 (2014) 9, 091304 .
[10] N. Kaloper and A. Padilla, Phys. Rev. D 90 (2014) 8, 084023 [Addendum-ibid. D 90 (2014) 10, 109901].

[11] N. Kaloper and A. Padilla, Phys. Rev. Lett. 114 (2015) 10, 101302

[12] N. Kaloper, A. Padilla, D. Stefanyszyn and G. Zahariade, Phys. Rev. Lett. 116 (2016) 5, 051302

[13] N. Kaloper, A. Padilla and D. Stefanyszyn, arXiv:1604.04000 [hep-th].

[14] N. Kaloper and A. Padilla, Phys. Rev. Lett. 118 (2017) no.6, 061303 [arXiv:1606.04958 [hep-th]].

[15] G. D'Amico, N. Kaloper, A. Padilla, D. Stefanyszyn, A. Westphal and G. Zahariade, arXiv:1705.08950 [hepth].

[16] C. Charmousis, E. J. Copeland, A. Padilla and P. M. Saffin, Phys. Rev. Lett. 108 (2012) 051101 [arXiv:1106.2000 [hep-th]].

[17] C. Charmousis, E. J. Copeland, A. Padilla and P. M. Saffin, Phys. Rev. D 85 (2012) 104040 [arXiv:1112.4866 [hep-th]].

[18] E. J. Copeland, A. Padilla and P. M. Saffin, JCAP 1212 (2012) 026 [arXiv:1208.3373 [hep-th]].

[19] N. Arkani-Hamed, S. Dimopoulos, N. Kaloper and R. Sundrum, Phys. Lett. B 480 (2000) 193 [hepth/0001197].

[20] C. Charmousis, E. Kiritsis and F. Nitti, arXiv:1704.05075 [hep-th].

[21] Y. Aghababaie, C. P. Burgess, S. L. Parameswaran and F. Quevedo, Nucl. Phys. B 680 (2004) 389 [hepth/0304256].

[22] C. P. Burgess and L. van Nierop, Phys. Dark Univ. 2 (2013) 1 [arXiv:1108.0345 [hep-th]].

[23] F. Niedermann and R. Schneider, JHEP 1602 (2016) 025 [arXiv:1508.01124 [hep-th]].

[24] F. Niedermann and R. Schneider, JHEP 1603 (2016) 130 [arXiv:1512.03800 [hep-th]].

[25] G. R. Dvali and G. Gabadadze, Phys. Rev. D 63 (2001) 065007 [hep-th/0008054].

[26] N. Kaloper and D. Kiley, JHEP 0705 (2007) 045 [hepth/0703190].

[27] S. L. Dubovsky and V. A. Rubakov, Phys. Rev. D 67, 104014 (2003) [hep-th/0212222].

[28] S. F. Hassan, S. Hofmann and M. von Strauss, JCAP 1101, 020 (2011) [arXiv:1007.1263 [hep-th]].

[29] F. Niedermann, R. Schneider, S. Hofmann and J. Khoury, Phys. Rev. D 91 (2015) no.2, 024002 [arXiv:1410.0700 [gr-qc]].

[30] L. Eglseer, F. Niedermann and R. Schneider, Phys. Rev. D 92 (2015) no.8, 084029 [arXiv:1506.02666 [gr-qc]].

[31] G. Dvali, S. Hofmann and J. Khoury, Phys. Rev. D 76, 084006 (2007) [hep-th/0703027 [hep-th]].

[32] N. Arkani-Hamed, S. Dimopoulos, G. Dvali and G. Gabadadze, hep-th/0209227.

[33] C. M. Will, Living Rev. Rel. 17 (2014) 4 [arXiv:1403.7377 [gr-qc]].

[34] G. Kallen, Helv. Phys. Acta 25 (1952) no.4, 417.

[35] H. Lehmann, Nuovo Cim. 11 (1954) 342.

[36] A. Adams, J. McGreevy and E. Silverstein, hepth/0209226.

[37] J. Khoury and A. Weltman, Phys. Rev. Lett. 93 (2004) 171104 [astro-ph/0309300].

[38] J. Khoury and A. Weltman, Phys. Rev. D 69 (2004) 044026 [astro-ph/0309411].

[39] A. I. Vainshtein, Phys. Lett. 39B (1972) 393.

[40] E. Babichev and C. Deffayet, Class. Quant. Grav. 30 
(2013) 184001 [arXiv:1304.7240 [gr-qc]].

[41] N. Kaloper, A. Padilla and N. Tanahashi, JHEP 1110 (2011) 148 [arXiv:1106.4827 [hep-th]].

[42] K. Hinterbichler and J. Khoury, Phys. Rev. Lett. 104 (2010) 231301 [arXiv:1001.4525 [hep-th]].

[43] K. Hinterbichler, J. Khoury, A. Levy and A. Matas, Phys. Rev. D 84 (2011) 103521 [arXiv:1107.2112 [astroph.COl].

[44] M. Porrati, Phys. Lett. B 498 (2001) 92 [hepth/0011152].

[45] S. Deser and A. Waldron, Phys. Lett. B 508 (2001) 347 [hep-th/0103255].

[46] S. Deser, M. Sandora and A. Waldron, Phys. Rev. D 87 (2013) no.10, 101501 [arXiv:1301.5621 [hep-th]].

[47] C. de Rham, K. Hinterbichler, R. A. Rosen and A. J. Tolley, Phys. Rev. D 88 (2013) no.2, 024003 [arXiv:1302.0025 [hep-th]].

[48] S. Deser and A. Waldron, Phys. Rev. D 87 (2013) 087702 [arXiv:1301.2238 [hep-th]].

[49] H. van Dam and M. J. G. Veltman, Nucl. Phys. B 22 (1970) 397.

[50] V. I. Zakharov, JETP Lett. 12 (1970) 312 [Pisma Zh. Eksp. Teor. Fiz. 12 (1970) 447].

[51] I. I. Kogan, S. Mouslopoulos and A. Papazoglou, Phys. Lett. B 503 (2001) 173 [hep-th/0011138].

[52] S. A. Appleby, A. De Felice and E. V. Linder, JCAP
1210 (2012) 060 [arXiv:1208.4163 [astro-ph.CO]].

[53] D. W. Dusedau and D. Z. Freedman, Phys. Rev. D 33 (1986) 389.

[54] J. Bros, Nucl. Phys. Proc. Suppl. 18B (1991) 22.

[55] H. Epstein, Pramana 78 (2012) 853.

[56] A. Higuchi, Nucl. Phys. B 282 (1987) 397.

[57] P. Breitenlohner and D. Z. Freedman, Annals Phys. 144 (1982) 249.

[58] L. Randall and R. Sundrum, Phys. Rev. Lett. 83 (1999) 4690 [hep-th/9906064].

[59] J. Garriga and T. Tanaka, Phys. Rev. Lett. 84 (2000) 2778 [hep-th/9911055].

[60] S. B. Giddings, E. Katz and L. Randall, JHEP 0003 (2000) 023 [hep-th/0002091].

[61] N. Arkani-Hamed, S. Dimopoulos, G. Dvali and G. Gabadadze, hep-th/0209227.

[62] G. Dvali, New J. Phys. 8 (2006) 326 hep-th/0610013.

[63] N. Arkani-Hamed, H. Georgi and M. D. Schwartz, Annals Phys. 305 (2003) 96 [hep-th/0210184].

[64] N. Kaloper, A. Padilla, P.M. Saffin and D. Stefanyszyn, Phys. Rev. D 91 (2015) no.4, 045017 [arXiv:1409.3243 [hep-th]].

[65] C. de Rham, S. Melville, A. J. Tolley and S. Y. Zhou, arXiv:1702.08577 [hep-th]. 